\title{
Numerical modelling of study the effect of the entrainment velocity, the number of Nusselt and the thickness of the non-convective zone on the stability of the pond solar
}

\author{
S. Gheraibia*1 ${ }^{1}$, A. Guesmia ${ }^{2}$ \\ ${ }^{1}$ Department of Physics, University 20 August 1955, Skikda, Algeria \\ ${ }^{2}$ Department of Mathematics, Laboratory LAMAHIS, University 20 August 1955, Skikda, Algeria
}

\begin{abstract}
In this paper, the effect of the entrainment velocity, the Nusselt number, and the thickness of the salinity gradient zone $(N C Z)$ on the stability of the solar pond are studied. The modelling equations of thermal energy and mass transfer in a salt gradient solar pond are discretized by finite difference methods in the transient regime. A new border condition applicable near the equilibrium of interface between the $(N C Z)$ and the $(L C Z)$ region is proposed. We take account of the effects of both turbulent entrainment and diffusion on the growth or erosion of the gradient zone $(N C Z)$. The obtained numerical results show an additional condition of solar pond's stability which links between the salinity gradient $(\Delta C)$ and the temperature gradient $(\Delta T)$ in the $(N C Z)$ region.
\end{abstract}

Mathematics Subject Classification (2020). 35K05, 76E06, 76E15

Keywords. solar pond, entrainment velocity, finite difference method

\section{Nomenclature}

$C$ : concentration of solution $\ldots\left(\mathrm{kgm}^{-3}\right)$

$\Delta C$ : concentration difference $\ldots \Delta C=C_{\max }-C_{\min }$

$C_{p}$ : specific heat of solution $\ldots\left(k_{J k g}{ }^{-1 \circ} C^{-1}\right)$

$D$ : diffusion coefficient $\ldots\left(m^{2} s^{-1}\right)$

$g$ : acceleration due to gravity $\ldots\left(m s^{-2}\right)$

$H$ : height of the solar pond ... $(m)$

$h_{c}$ : convective heat transfer coefficient $\ldots\left(W m^{-2 \circ} C^{-1}\right)$

$L$ : length of the non-convective zone ... $(m)$

$L C Z$ : lower convective zone

$N C Z$ : non-convective zone

$U C Z$ : upper convective zone

$L_{e}$ : Lewis number

$P_{r}$ : Prandt number

*Corresponding Author.

Email addresses: ghearibia2sabo@gmail.com, s.gheraibia@uni-skikda.dz (S. Gheraibia), guesmiasaid@yahoo.fr, a.guesmia@uni-skikda.dz (A. Guesmia)

Received: 26.10.2020; Accepted: 30.01.2021 
$S_{c}:$ Schmidt number

$q_{0}$ : solar radiation that penetrates the free-surface after reflection

from pond's surface ... $\left(W m^{-2}\right)$

$q(z)$ : quantity of solar radiation at depth $z \ldots\left(W^{-2}\right)$

$R_{a T}$ : thermal Rayleigh number

$R_{a I}$ : internal Rayleigh number

$R_{a S}$ : solutal Rayleigh number

$N_{u}$ : Nusselt number

$t$ : time ... $(s)$

$\Delta T:$ temperature difference $\ldots \Delta T=T_{\max }-T_{\min }$

$T$ : temperature ... $\left({ }^{\circ} \mathrm{C}\right)$

$T_{a}$ : ambient temperature $\ldots\left({ }^{\circ} \mathrm{C}\right)$

$U_{e}$ : dimensionless entrainment velocity $\ldots\left(m^{-1}\right)$

$u_{*}$ : convection velocity $\ldots m s^{-1}$

$u_{e}$ : entrainment velocity $\ldots m s^{-1}$

$R i$ : Richardson number

$z$ : Cartesian coordinate ... $(m)$

$Z$ : dimensionless Cartesian coordinate

\section{Greek symbols}

$\alpha$ : thermal diffusivity $\ldots\left(m^{2} s^{-1}\right)$

$\rho$ : density $\left(\mathrm{kgm}^{-3}\right)$

$\beta_{T}$ : thermal expansion coefficient $\ldots\left(K^{-1}\right)$

$\beta_{S}$ : solutal expansion coefficient $\ldots\left(\mathrm{m}^{3} \mathrm{~kg}^{-1}\right)$

$\lambda_{w}$ : water thermal conductivity $\ldots\left(W m^{-1} K^{-1}\right)$

$\nu$ : cinematic viscosity $\ldots\left(m^{2} s^{-1}\right)$

$\mu$ : dynamic viscosity $\ldots\left(\mathrm{kgm}^{-1} \mathrm{~s}^{-1}\right)$

$\chi$ : dimensionless concentration

$\theta$ : dimensionless temperature

$\theta_{r}$ : angle of reflection at the pond's surface $\ldots\left(^{\circ}\right)$

$\tau$ : dimensionless time of heating

$\varepsilon$ : absorption coefficient ... $\left(m^{-1}\right)$

$\Phi_{j}$ : dimensionless absorption coefficient $\ldots\left(\Phi_{j}=\varepsilon_{j} H\right)$

$\gamma$ : fraction of radiation

\section{Introduction}

A solar pond is a pool of saltwater that collects and stores solar thermal energy. It is constituted by three well-defined zones (see Figure 1); the upper (UCZ) and lower (LCZ) convective zones, where temperature can be considered as uniform, the gradient flank zone (GZ) or (NCZ) characterized by a concentration and a temperature gradient.

The layers of salt solutions increase in concentration (and therefore density) with depth. Solar ponds provide the simplest technique for transforming the sun's energy into a solar energy which can be extracted for different purposes. The bottom of the pond is warmed to extremely high temperatures, it can reach about $90^{\circ} \mathrm{C}$ [7].

The stability of the solar pond is related to the gradient zone stabilization which is affected by many different factors like the double-diffusive convection between the low convective zone (LCZ) and the non-convective zone (NCZ). This leads to a difference in the diffusion rate of salt and temperature which produces the loss of energy. The erosion and the growth of the gradient zone depend on the strength of convective velocities in the 
mixed layers, the values of gradient temperature, and the gradient salinity in the (NCZ) region. The stationary interface, for zero entrainment velocity, is known as the equilibrium condition. Inside the field, it is difficult to notice the movement of the interface, about 1 centimetre $(\mathrm{cm})$ per week. In the laboratory study of Xu [25], the interface movement within this limit is considered as in equilibrium.

Many studies of solar ponds, including experimental, analytical, and numerical investigations, have received much attention from numerous researchers. Among them, we can cite the works of Ould Dah et al. [16], who studied experimentally and numerically the efficiency and effectiveness of a mini solar pond. They developed a one-dimensional model to study the thermal and solute behavior in the mini solar pond. In their research work, the efficiency of the mini solar pond could be improved considerably by using a new method of heat extraction from the (NCZ). Bansal et al. [2], solved the one-dimensional equation of heat conduction with fluid internal heating due to the solar radiation absorption. Wang et al. [24], studied numerically the nonlinear dynamic behavior of the non-convective zone in a salt gradient solar pond. In their work, the nonlinear results indicate that the linear stability analysis leans to a larger upper boundary in the oscillatory regions. Zangrando and Fernando [26] studied the rate of entrainment and detrainment and the conditions for equilibrium for diffusive boundaries of a double-diffusive fluid layer.

The system consists of a stably stratified layer overlying a mixed, turbulently convecting layer, which is representative of solar pond situations and others. Most of them have shown that the performance and the efficiency of solar ponds depend on the stability of the gradient layer. In very recent works, the authors have studied the hydrodynamic, heat, and mass transfer behaviors of a salinity gradient solar pond in transient regime. The results have shown, in particular, that the internal Rayleigh number, the buoyancy ratio, and the aspect ratio have an important effect, respectively, on the thermal performance of the pond, on the stability, and on the distribution of temperature, and velocity fields in the salinity gradient solar pond. In the present paper, we aim to study numerically the temporal evolution of the temperature and concentration distributions in a one dimensional insulated salinity gradient solar pond under the influence of the entrainment velocity and the number of Nusselt. The resolution of energy, and mass transfer equations are conducted using finite-difference discretization method in transient regime.

\section{Mathematical model for the salt gradient solar pond}

The solar pond is modeled with a two-dimensional rectangular enclosure, as shown in Figure 1. Some assumptions have been made to simplify the analysis of the hydrodynamic, thermal, and solute behavior of solar basins:

1- The amount of solar radiation absorbed at depth $\mathrm{z}$ is given by Rabl and Nielsen's model [17] and is assumed to be constant and has an average value in the transient system.

2- The change of velocity, temperature, and concentration along the y-direction is negligible.

3- Both the vertical and the bottom walls of the pond are well insulated and impermeable. 


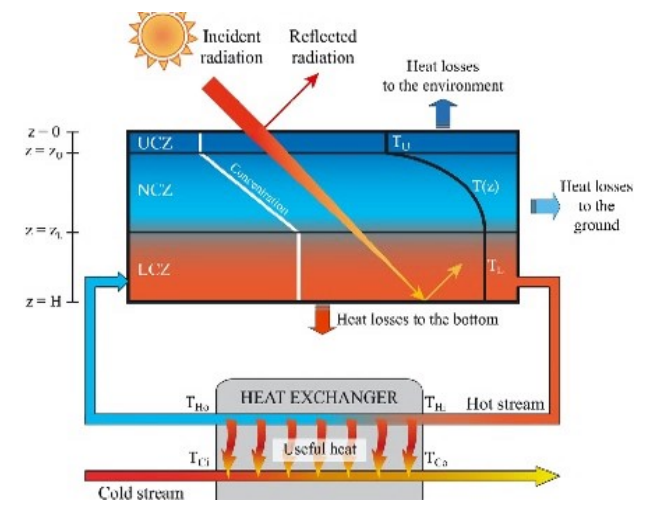

Figure 1. Schematic view of the insulated salt gradient solar pond [10].

In this work, under the above assumptions, the transient heat, and mass transfer process in the solar pond with internal heating of the fluid due to solar radiation absorption are governed by thermal energy and mass transfer equations in $(N C Z)$ region:

- Equations of thermal energy:

$$
\frac{d T}{d t}-\alpha \frac{d^{2} T}{d z^{2}}=\frac{1}{\rho c_{p}} \frac{d q(z)}{d z}
$$

with initial condition

$$
T(t, z)=T_{a} \quad \text { if } t=0 \quad \forall z \in\left[z_{N}, z_{L}\right],
$$

and boundary condition

$$
\frac{d T}{d t}+u_{e} \frac{d T}{d z}=\frac{h_{c}}{\rho c_{p}}\left(T-T_{a}\right)-\frac{1}{\rho c_{p}} \frac{d q(z)}{d z} \quad \forall t \text { and } \quad z=z_{L}
$$

- Equations of mass transfer:

with initial condition

$$
\frac{d C}{d t}-D \frac{d^{2} C}{d z^{2}}=0
$$

$$
C(t, z)=\Delta C\left(\frac{z-z_{N}}{z_{L}-z_{N}}\right)+C_{\min } \quad \text { if } \quad t=0 \quad \forall z \in\left[z_{N}, z_{L}\right]
$$

and boundary condition

$$
\begin{aligned}
& \frac{d C}{d t}+\left(u_{e}+\frac{D}{z_{N}}\right) \frac{d C}{d z}=0 \quad \forall t \quad \text { and } \quad z=z_{N} \\
& \frac{d C}{d t}+\left(u_{e}+\frac{D}{H-z_{L}}\right) \frac{d C}{d z}=0 \quad \forall t \quad \text { and } \quad z=z_{L}
\end{aligned}
$$

The quantity, $q(z)$, of solar radiation at different depths of salty water in the solar pond can be expressed as [17]:

$$
q(z)=k q_{0} \sum \gamma_{j} \exp \left[\frac{-\varepsilon_{j}}{\cos \theta_{r}}(z)\right]
$$

where $q_{0}$ represents the solar radiation that penetrates the free-surface of the solar pond, which is given by

$$
q_{0}=(1-r) q_{i}
$$


where $q_{i}$ is the incident solar radiation that reaches the free-surface of the solar pond. Here $r$ is the reflectance of solar radiation at the free-surface of the pond.

This coefficient is obtained from [17],

$$
r=0.5\left[\frac{\tan ^{2}\left(\theta_{i}-\theta_{r}\right)}{\tan ^{2}\left(\theta_{i}+\theta_{r}\right)}+\frac{\sin ^{2}\left(\theta_{i}-\theta_{r}\right)}{\sin ^{2}\left(\theta_{i}+\theta_{r}\right)}\right],
$$

where $\theta_{i}$ is the angle of incidence, $\theta_{r}$ the angle of reflection, $k$ a coefficient due to the effects of salt concentration, propagation of radiation in the layers of salty water, and turbidity of water on the reduction of solar radiation.

For the present study,

$$
\theta_{i}=60^{\circ}, \theta_{r}=40.5^{\circ}, k=0.85 \text { and } r=0.06 \quad[26] .
$$

The tow coefficients $\gamma_{j}$ and $\varepsilon_{j}$ are dependent on the absorption of the particular wavelengths range of the arriving solar radiations. These constants $\gamma_{j}$ and $\varepsilon_{j}$ are given in Table $1,[3]$.

Table 1. The constants $\gamma_{j}$ and $\varepsilon_{j}$

\begin{tabular}{|c|c|c|c|c|}
\hline wavelength & $\mathbf{0 . 2 - 0 . 6}$ & $\mathbf{0 . 6 - 0 . 7 5}$ & $\mathbf{0 . 7 5 - 0 . 9}$ & $\mathbf{0 . 9 - 1 . 2}$ \\
\hline$\gamma$ & 0.237 & 0.193 & 0.167 & 0.179 \\
$\varepsilon$ & 0.032 & 0.45 & 3 & 3.5 \\
\hline
\end{tabular}

Finally, equation $q(z)$ is expressed as follows:

$$
q(z)=0.85 q_{0} \sum \gamma_{j} \exp \left[\frac{-\varepsilon_{j}}{0.76}(z)\right] .
$$

Then the rate of energy generation per volume unit in a fluid layer, which results from the solar radiation absorption by salty water, is given by [23]

$$
f(z)=\frac{1}{\rho c_{p}} \frac{d q(z)}{d z}=0.85 \frac{q_{0}}{\rho c_{p}} \sum \frac{\gamma_{j} \varepsilon_{j}}{0.76} \exp \left[\frac{-\varepsilon_{j}}{0.76}(z)\right] .
$$

In order to simplify the study, the heat field and salt concentration of a salinity gradient zone (GZ) can be described by a set of differential non-dimensional equations by using the following dimensionless parameters.

$$
\begin{gathered}
Z=z / H, \quad U_{e}=u_{e} /(\alpha / H), \quad \tau=\frac{t}{\left(H^{2} / \alpha\right)}, \quad \theta=\frac{\left(T-T_{a}\right)}{\Delta T}, \\
\chi=\frac{\left(C-C_{\min }\right)}{\Delta C}, \quad R_{a T}=\frac{g \beta_{T} \Delta T H^{3}}{(\alpha \nu)}, \quad R_{a I}=\frac{g \beta_{T} H^{4} q_{0}}{\left(\lambda_{w} \alpha \nu\right)}, \quad R_{a s}=\frac{g \beta_{C} \Delta C H^{3}}{(\alpha \nu)}, \\
\operatorname{Pr}=\nu / \alpha, \quad L e=\alpha / D, \quad S_{c}=\nu / D, \quad N_{u}=\frac{L h_{c}}{\lambda_{w}} .
\end{gathered}
$$


- Energy equation with the initial and boundary conditions:

$(N C Z)$ zone:

$$
\left(P_{N C Z}\right)\left\{\begin{array}{c}
\frac{\partial \theta(\tau, Z)}{\partial \tau}=\frac{\partial^{2} \theta(\tau, Z)}{\partial Z^{2}}+f(Z) \quad(\tau, Z) \in\left[0, \tau_{\max }\right] \times\left[Z_{N}, Z_{L}\right] \\
\theta(0, Z)=\theta_{a b} \\
\theta\left(\tau, Z_{N}\right)=\theta_{a b} \\
\left\{\begin{array}{c}
\frac{\partial \theta\left(\tau, Z_{L}\right)}{\partial \tau}+U_{e} \frac{\partial \theta\left(\tau, Z_{L}\right)}{\partial Z}=N_{u} \theta\left(\tau, Z_{L}\right)-f\left(Z_{L}\right) \quad \tau \in\left[0, \tau_{\max }\right] \\
\theta\left(0, Z_{L}\right)=\theta_{a b}
\end{array}\right.
\end{array}\right.
$$

Thus, the dimensionless equation of $f(z)$ is given by

$$
f(Z)=0.85 \frac{R_{a I}}{R_{a T}} \sum \frac{\gamma_{j} \varepsilon_{j}}{0.76} \exp \left[\frac{-\phi_{j}}{0.76}(Z)\right],
$$

where $\theta_{a b}$ is dimensionless ambient temperature.

- Mass transfer equation with the initial and boundary conditions:

$N C Z$ zone:

$$
\left(P_{N C Z}\right)\left\{\begin{array}{c}
\left.\frac{\partial \chi(t, Z)}{\partial \tau}=\frac{p_{r}}{s_{c}} \frac{\partial^{2} \chi(t, Z)}{\partial z^{2}} \quad Z \in\right] Z_{N}, Z_{L}[ \\
\chi(0, Z)=\frac{\left(Z-Z_{N}\right)}{\left(Z_{L}-Z_{N}\right)} \\
\frac{\partial \chi\left(\tau, Z_{N}\right)}{\partial \tau}=\frac{1}{Z_{N}} \frac{p_{r}}{s_{c}} \frac{\partial \chi\left(\tau, Z_{N}\right)}{\partial Z} \\
\frac{\partial \chi\left(\tau, Z_{L}\right)}{\partial \tau}+U_{e} \frac{\partial \chi\left(\tau, Z_{L}\right)}{\partial Z}=-\frac{1}{\left(1-Z_{L}\right)} \frac{p_{r}}{s_{c}} \frac{\partial \chi\left(\tau, Z_{L}\right)}{\partial Z}
\end{array}\right.
$$

\section{Numerical method}

The equations which describe the phenomena of transfer of temperature and salt concentration in the solar pond are solved numerically by various methods as the homotopy perturbation method [1,19-22], the finite volume method [11-13], and the finite difference method. In our study we used the finite difference method.

\subsection{The finite difference method}

Let $h>0$ be the mesh size of discretization in height. We take the grid points $Z_{i}=$ $Z_{i-1}-h, i \in\{1,2, \ldots\}$ with $Z_{0}=Z_{U}, Z_{m}=Z_{L}$.

Let $\Delta \tau>0$ be the mesh size of discretization in time. We take the grid points $\tau^{n}=$ $\tau^{n-1}+\Delta \tau, n \in\{1,2, \ldots, P\}$ with $\tau^{0}=0$.

We define the approximations $\left(\tau^{n}, Z_{i}\right)$ in temperature by $\theta_{i}^{n}=\theta\left(\tau^{n}, Z_{i}\right)$.

For solving the first numerical problem we use the implicit scheme which is unconditionally stable and consistent with the truncation error $O\left((h)^{2}+(\Delta \tau)^{1}\right)[15]$ : 


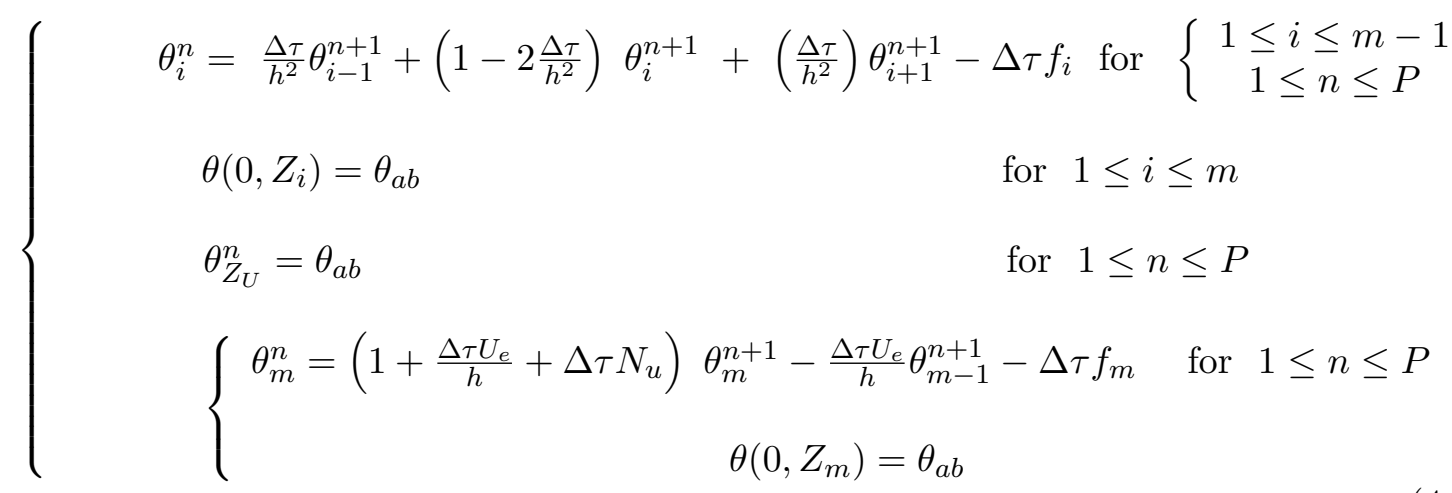

The problem can be written in the matrix form as:

$$
\overrightarrow{\theta^{n}}=[A] \overrightarrow{\theta^{n+1}}+\overrightarrow{b^{n+1}} \Longrightarrow\left\{\begin{array}{cc}
\overrightarrow{\theta^{n+1}}=[A]^{-1} \overrightarrow{\theta^{n}}-[A]^{-1} \overrightarrow{b^{n+1}} & \text { for } 0 \leq n \leq P \\
\overrightarrow{\theta^{0}}=\overrightarrow{\theta_{a b}} &
\end{array}\right.
$$

where $[A]$ is an $m \times m$ matrix and $\overrightarrow{b^{n}}, \overrightarrow{\theta^{n}}$ are two vectors of dimension $m$ given by the following expressions:

$$
[A]=\left[\begin{array}{ccccccc}
\alpha & \beta & 0 & \ldots & \ldots & \ldots & 0 \\
\gamma & \alpha & \beta & 0 & \ddots & \ddots & 0 \\
0 & \ddots & \ddots & \ddots & \ddots & \ddots & \vdots \\
\vdots & \ddots & \ddots & \ddots & \ddots & \ddots & \vdots \\
\vdots & \ddots & \ddots & \ddots & \ddots & \ddots & 0 \\
\vdots & \ddots & \ddots & \ddots & \gamma & \alpha & \beta \\
0 & \ldots & \ldots & \ldots & 0 & \lambda & \varpi
\end{array}\right] \quad \text { where } \quad\left\{\begin{array}{c}
\alpha=\left(1-2 \frac{\Delta \tau}{h^{2}}\right) \\
\beta=\frac{\Delta \tau}{h^{2}} \\
\gamma=\frac{\Delta \tau}{h^{2}} \\
\varpi=\left(1+\left(\frac{\Delta \tau}{h} U_{e}\right)+N_{u} \Delta \tau\right) \\
\lambda=-\left(\frac{\Delta \tau}{h} U_{e}\right)
\end{array}\right.
$$

and

$$
\overrightarrow{\theta^{n}}=\left[\begin{array}{c}
\theta_{1}^{n} \\
\theta_{2}^{n} \\
\vdots \\
\theta_{m-1}^{n} \\
\theta_{m}^{n}
\end{array}\right], \quad \overrightarrow{b^{n}}=\left[\begin{array}{c}
-\Delta \tau f_{1}+\gamma \theta_{0}^{n} \\
-\Delta \tau f_{2} \\
\vdots \\
-\Delta \tau f_{m-1} \\
-\Delta \tau f_{m}
\end{array}\right], \quad \text { and } \overrightarrow{\theta^{0}}=\theta_{a b}\left[\begin{array}{c}
1 \\
1 \\
\vdots \\
1 \\
1
\end{array}\right]
$$

We define the approximations $\left(\tau^{n}, Z_{i}\right)$ in concentration by $\chi_{i}^{n}=\chi\left(\tau^{n}, Z_{i}\right)$. For solving the second numerical problem, we use the implicit scheme: 


$$
\left\{\begin{array}{c}
\left(1+\left(\frac{\Delta \tau}{Z_{0}} \frac{p r}{h s c}\right)\right) \chi_{0}^{n+1}-\left[\left(\frac{\Delta \tau}{Z_{0}} \frac{p r}{h s c}\right)\right] \chi_{1}^{n+1}=\chi_{0}^{n} \quad i=0 \\
-\left(\frac{\Delta \tau p r}{h^{2} s c}\right) \chi_{i-1}^{n+1}+\left(1+2 \frac{\Delta \tau p r}{h^{2} s c}\right) \chi_{i}^{n+1}-\frac{\Delta \tau p r}{h^{2} s c} \chi_{i+1}^{n+1}=\chi_{i}^{n} \quad 1 \leq i \leq m-1 \\
-\left(\frac{\Delta \tau}{h} U_{e}+\frac{\Delta \tau}{1-Z_{m}} \frac{p r}{h s c}\right) \chi_{m-1}^{n+1}+\left(1+\frac{\Delta \tau}{h} U_{e}+\frac{\Delta \tau}{\left(1-Z_{m}\right)} \frac{p r}{h s c}\right) \chi_{m}^{n+1}=\chi_{m}^{n} \quad i=m \\
\chi_{i}^{0}=\frac{\left(Z_{m}-Z_{i}\right)}{\left(Z_{m}-Z_{0}\right)} \quad 0 \leq i \leq m \quad \text { initial condition vector }
\end{array}\right.
$$

The problem can be written in the matrix form as:

$$
\overrightarrow{\chi^{n}}=[A] \overrightarrow{\chi^{n+1}} \Longrightarrow\left\{\begin{array}{c}
\overrightarrow{\chi^{n+1}}=[A]^{-1} \overrightarrow{\chi^{n}} \\
\overrightarrow{\chi^{0}}=\frac{1}{\left(Z_{m}-Z_{0}\right)}\left(Z_{m} \vec{I}-\vec{Z}\right)
\end{array} \quad \text { for } \quad 0 \leq n \leq P\right.
$$

where $[A]$ is an $(m+1) \times(m+1)$ matrix and $\vec{I}$ (Identity), $\overrightarrow{\chi^{n}}$ are two vectors of dimension $(m+1)$ given by the following expressions:

$$
[A]=\left[\begin{array}{ccccccc}
\alpha & \beta & 0 & \ldots & \ldots & \ldots & 0 \\
\gamma & \eta & \kappa & 0 & \ddots & \ddots & 0 \\
0 & \ddots & \ddots & \ddots & \ddots & \ddots & \vdots \\
\vdots & \ddots & \ddots & \ddots & \ddots & \ddots & \vdots \\
\vdots & \ddots & \ddots & \ddots & \ddots & \ddots & 0 \\
\vdots & \ddots & \ddots & \ddots & \gamma & \eta & \kappa \\
0 & \ldots & \ldots & \ldots & 0 & \lambda & \varpi
\end{array}\right] \quad \text { where } \quad\left\{\begin{array}{c}
\alpha=\left(1+\left(c_{1} * c_{2}\right)\right) \\
\beta=-\left(\left(c_{1} * c_{2}\right)\right) \\
\gamma=-\left(\frac{c_{1}}{h}\right) \\
\eta=\left(1+2 \frac{c_{1}}{h}\right) \\
\kappa=-\frac{c_{1}}{h} \\
\\
\end{array}\right.
$$

with

and

$$
w=\frac{\Delta \tau}{h}, \quad v=\frac{P_{r}}{S_{c}}, \quad c_{1}=w * v, \quad c_{2}=\frac{1}{Z_{0}}, \quad c_{N 3}=w U_{e}, \quad c_{4}=\frac{1}{\left(1-Z_{m}\right)},
$$

$$
\overrightarrow{\chi^{n+1}}=\left[\begin{array}{c}
\chi_{1}^{n+1} \\
\chi_{2}^{n+1} \\
\vdots \\
\chi_{m-1}^{n+1} \\
\chi_{m}^{n+1}
\end{array}\right], \quad \overrightarrow{\chi^{0}}=\left[\begin{array}{c}
0 \\
\frac{-1}{\left(Z_{m}-Z_{0}\right)}\left(Z_{0}-Z_{1}\right) \\
\vdots \\
\frac{-1}{\left(Z_{m}-Z_{0}\right)}\left(Z_{0}-Z_{m-1}\right) \\
1
\end{array}\right]
$$




\section{Graphics results}

Here, we present graphics results of transient regimes to understand the thermal and solute characteristics induced in a salt gradient solar pool better with controlling the parameters that define fluid flow, heat, and mass transfer in the (NCZ) region. The discussion of the graphs is given in Section 5 .
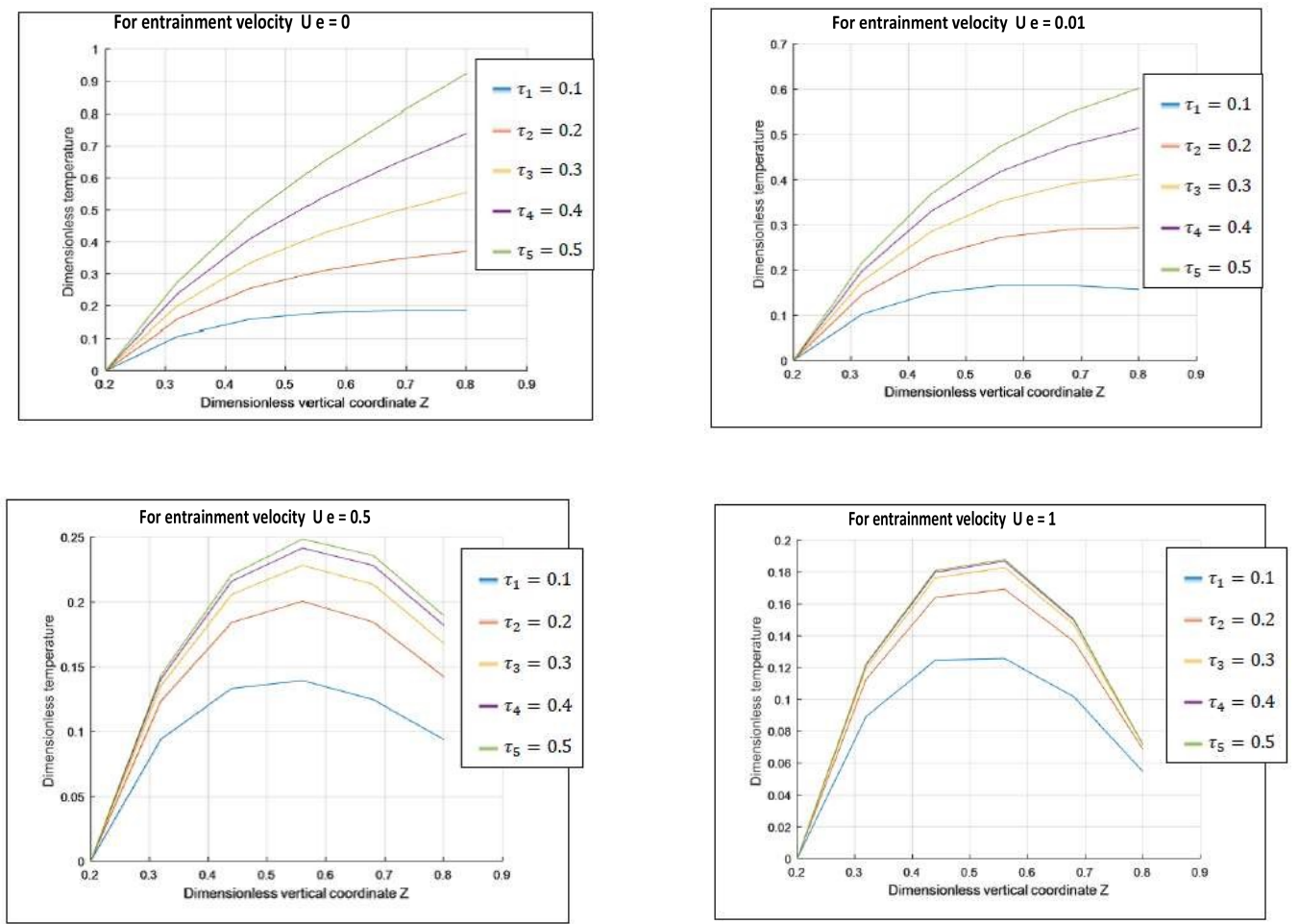

Figure 2. Effect of dimensionless entrainment velocity $U_{e}$ on the dimensionless temperature in $(N C Z)$ region $\tau=0.5$ and for $R_{a T}=10^{7}, R_{a I}=2 \times 10^{8}, N_{u}=$ 0.01 .

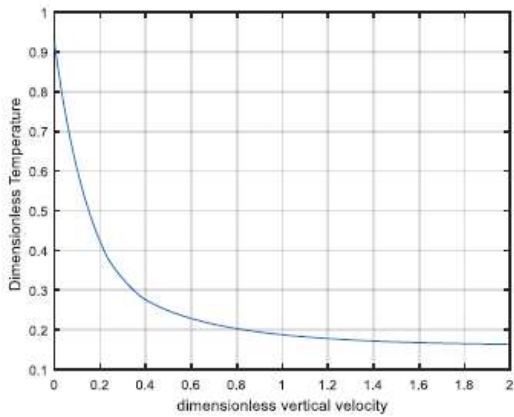

Figure 3. Variation of dimensionless temperature with dimensionless entrainment velocity in $(N C Z)$ region for $Z_{L}=0.8$. 

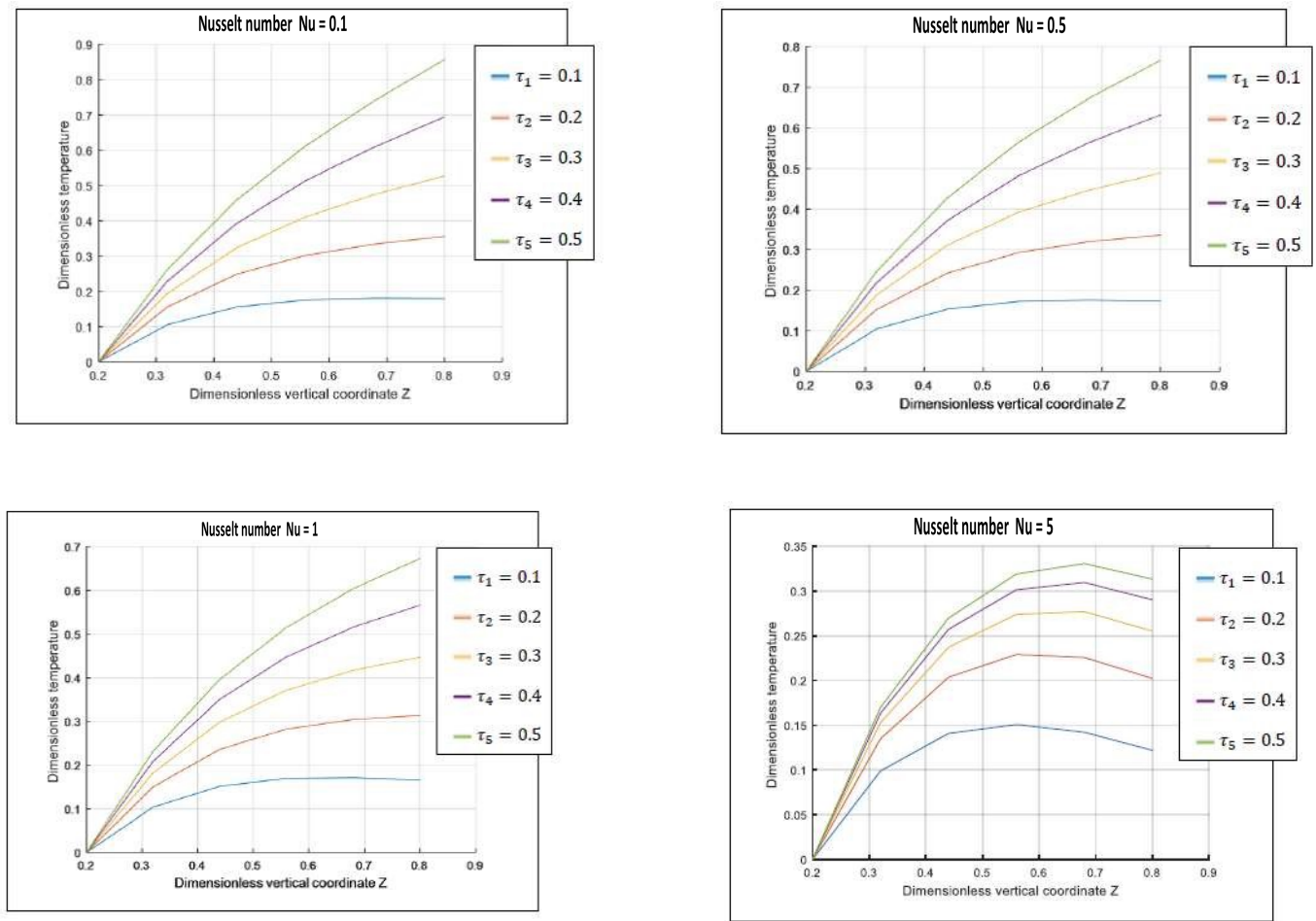

Figure 4. Effect of Nusselt number on the dimensionless temperature in $(N C Z)$ for $U_{e}=0.01$.

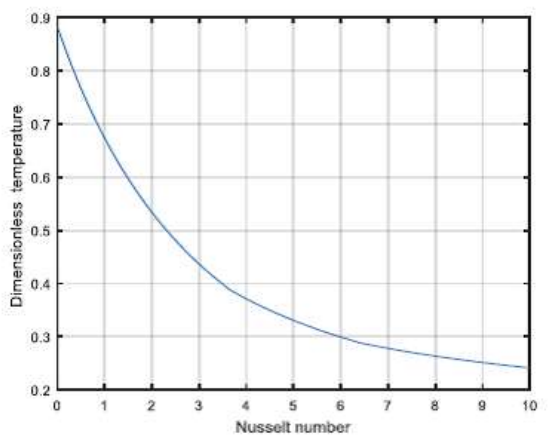

Figure 5. Variation of dimensionless temperature with Nusselt number in $N C Z$ region for $Z_{L}=0.8$ 

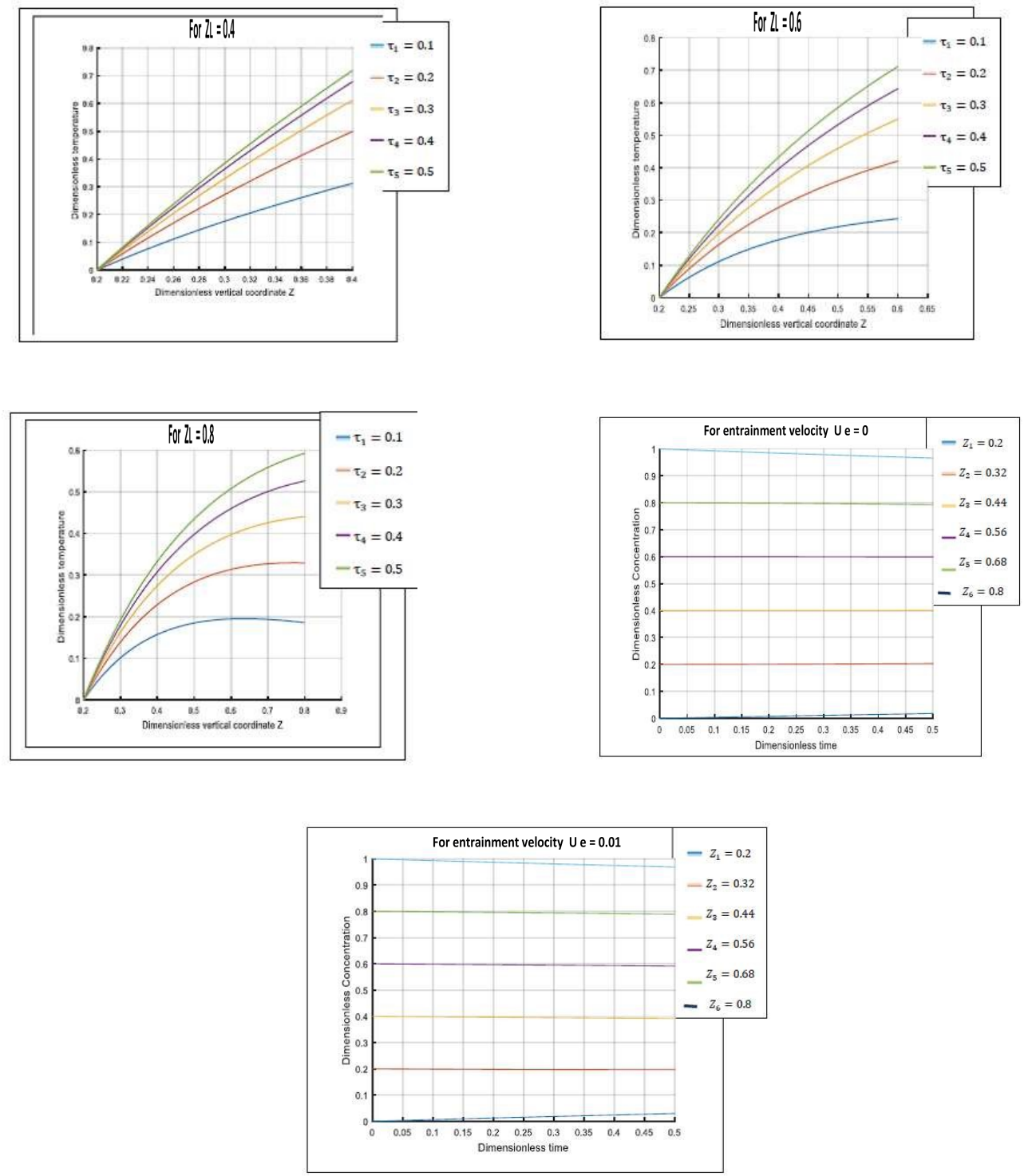

Figure 6. Evolution of the dimensionless temperature with the thickness of $(N C Z)$ region at $\tau=0.5$ and for $R_{a T}=10^{7}, R_{a I}=2 \times 10^{8}, U_{e}=0.01, N_{u}=0.1$, $Z_{U}=0.2$. 

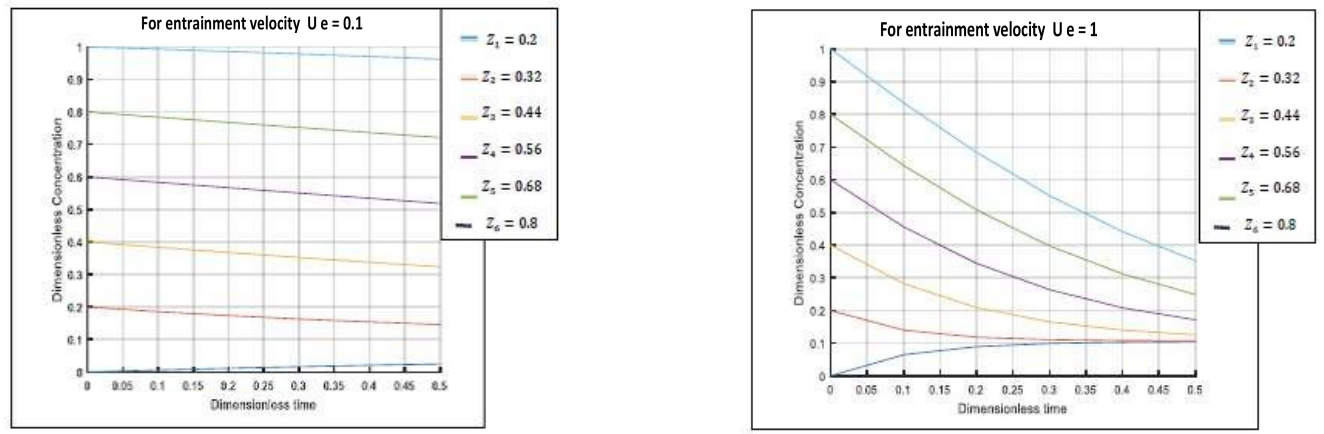

Figure 7. Effect of dimensionless entrainment velocity $U_{e}$ on the dimensionless concentration in $(N C Z)$ region $\tau=0.5$ and for $P_{r}=6, S c=10^{3}, N_{u}=0.01$.
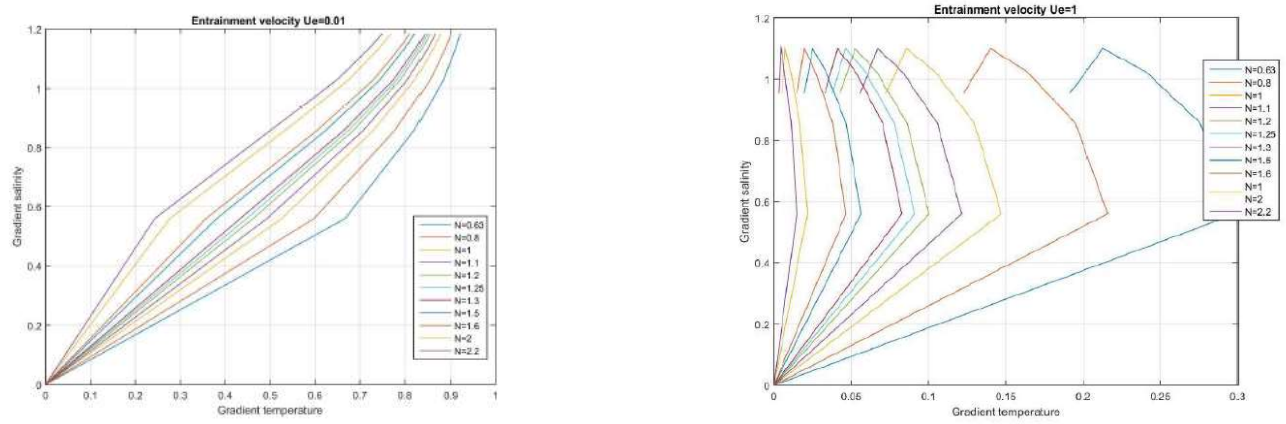

Figure 8. The variation of gradient salinity with gradient temperature in $(N C Z)$ region for $\tau=0.5$ and for $R_{a T}=10^{7}, R_{a I}=2 \times 10^{8}, P_{r}=6, S c=10^{3}, N_{u}=0.01$.

\section{Discussion}

In this section, we discuss the graphs given in the previous section. Here numerical results are performed with the following constant parameters: $R_{a T}=10^{7}, R_{a I}=2 \times$ $10^{8}, S c=1000$ and $P_{r}=6$.

\subsection{Effect of dimensionless entrainment velocity $\left(U_{e}\right)$ on the stability of solar pond}

The Figures 2 and 3 show that for small values of entrainment velocity $\left(U_{e}\right)$, the dimensionless temperature reaches its highest point. In this case, the (NCZ) region remains stable due to the equilibrium of the interface between both (NCZ) and (LCZ) regions. These last results are confirmed by many several experimental works $[4,6,18]$ under typical conditions on solar ponds (see Tables 2 and 3): 
Table 2. Typical conditions on solar ponds

\begin{tabular}{|c|c|c|}
\hline$u_{e}=c u_{*} R i^{-n}$ & $R i=\frac{g \Delta \rho z_{L}}{\rho u_{*}^{2}}$ & $c=0.2, n=1$ \\
\hline$u_{e}=0.11 \times 10^{-6} m s^{-1}$ & $R i=452$ & $u_{*}=2.5 \times 10^{-4} m s^{-1}$ \\
\hline
\end{tabular}

Table 3. Numerical data

\begin{tabular}{|c|c|}
\hline$U_{e}=u_{e} /(\alpha / H)$ & $\alpha \simeq 10^{-5} m^{2} s^{-1}$ \\
\hline$u_{e}=0.01$ & $H=1 m$ \\
\hline
\end{tabular}

Undoubtedly, the Reynolds number is so small that we consider the saltwater just as the Stokes fluid [9]. Generally, we can write,

$$
R_{e}=\frac{u_{e} z_{L}}{\nu H} \simeq \frac{U_{e}}{p_{r}} \simeq \frac{0.01}{6} \ll 1 .
$$

On the other hand, for a considerable entrainment velocity $\left(U_{e}\right)$, the value of the dimensionless temperature has shown a steady decline, owing to the dynamics erosion of lower interfaces.

The graph from the Figure 7 illustrates the effect of the dimensionless entrainment velocity $\left(U_{e}\right)$ on the dimensionless concentration in the (NCZ) region. Overall, the concentration decreases when the entrainment velocity $\left(U_{e}\right)$ rises and takes a considerable value. This clearly due to the spread of the salt in water from the bottom of the pond to the top [8]. In general, this graph highlights the evidence that the (NCZ) zone is inefficient under these conditions.

\subsection{Effect of the Nusselt number on the stability of solar pond}

The graphs given in the Figures 4 and 5 present data on the effect of Nusslet number on the distribution of temperature in a solar pond at $\tau=0.5$ and $U_{e}=0.01$. We notice that when $0 \leq N u \leq 1$, the dimensionless temperature reaches its highest value. Therefore, this provides evidence that the convective heat transfer coefficient $(h c)$ rises compared with the water thermal conductivity coefficient, which means falling in heat loss by convection phenomena.

According to the experimental relation between Nusslet number and Reynolds number [5],

$$
N u=0.037 \times R_{e}^{\frac{4}{5}} \times p_{r}^{\frac{1}{3}} .
$$

By using (4.3), we obtain the following result:

$$
N u=0.037 \times U_{e}^{\frac{4}{5}} \times p_{r}^{\frac{-2}{3}} \Rightarrow N u \ll 1 .
$$

\subsection{Effect of thickness of the salinity gradient zone $(N C Z)$ on the stability of solar pond}

The graph given in Figure 6 shows that the growth of the thickness of the (NCZ) region leads to an increase of the dimensionless temperature. One reason for this could be that the decrease of radiation solar energy with the depth of the fluid layer, conversely, at the same time, there is a marked reduction of incoming heat losses. 


\subsection{The condition of stability}

The experimental expression for the equilibrium condition in the gradient zone is given in [14],

$$
\frac{d C}{d z}=28\left(\frac{d T}{d z}\right)^{0.63}
$$

It seems that the relation between gradient salinity and gradient temperature is only a linear connection.

The graph given in Figure 8 shows that for small entrainment velocity, the salinity gradient varies linearly with the temperature gradient. This linear relation depends on the power value $(N)$ of the gradient temperature $\left(\frac{d T}{d z}\right)^{N}$. We notice that in the case of $1 \preceq N \preceq 1.5$, the relation keeps linear. However, it fluctuates markedly when $N \notin[1,1.5]$.

In the general case, we can write the stability relation of the solar pond as

$$
\frac{d C}{d z}=\alpha\left(\frac{d T}{d z}\right)^{N}
$$

Here $\alpha$ is a constant and it depends on the property of salty water.

On the other hand the dimensionless equation is given as

$$
\begin{aligned}
\frac{\Delta C}{H} \frac{d \chi}{d Z}=\alpha\left(\frac{\Delta T}{H} \frac{d \theta}{d Z}\right)^{N} & \Leftrightarrow \frac{d \chi}{d Z}=\frac{\alpha H^{1-N}(\Delta T)^{N}}{\Delta C}\left(\frac{d \theta}{d Z}\right)^{N} \\
& \Leftrightarrow \frac{d \chi}{d Z}=\alpha \xi\left(\frac{d \theta}{d Z}\right)^{N},
\end{aligned}
$$

with

$$
\xi=\frac{H^{1-N}(\Delta T)^{N}}{\Delta C}=\gamma / \alpha
$$

where $\gamma$ is the slope of the curve determined by $\frac{d \chi}{d Z}=f\left(\left(\frac{d \theta}{d Z}\right)^{N}\right)$, and for

$$
H=1 \Rightarrow \frac{(\Delta T)^{N}}{\Delta C}=\gamma / \alpha
$$

Finally, we deduce a new equilibrium condition in the gradient zone between $\Delta T$ and $\Delta C$ given as

$$
\begin{aligned}
(\Delta T)^{N} & =(\gamma / \alpha) \Delta C \\
\Rightarrow \Delta C & =(\alpha / \gamma)(\Delta T)^{N}
\end{aligned}
$$

For $U e=0.01, N=1.25$, and $\gamma=1.38$, we get $\Delta C=(\alpha / 1.38)(\Delta T)^{1.25}$.

\section{Conclusion}

In this work, we have studied the problem of the heat, mass transfer, and the condition of the stability in salt gradient solar pond under new boundary conditions. Moreover, this study has shown the importance of both Nusslet number and (NCZ) thickness in preventing the loss of the heat in the solar pond ((NCZ) region) by the convection phenomena. As has been noted, the small values of the entrainment velocity has also effect on the stability of the solar pond. Furthermore, the conditions of stability are linked to the linear relation between the salinity and the temperature gradient. In the end, what makes our work different and more interesting is that we have found an interval of exponential values of gradient temperature which makes the solar pond more stable. 


\section{References}

[1] Z. Ayati and J. Biazar, On the convergence of the homotopy perturbation method, J. Egyptian Math. Soc. 23 (2), 424-428, 2015.

[2] P.K. Bansal and N.D. Kaushika, Salt gradient stabilized solar pond collector, Energy Convers. Manag. 21, 81-95, 1981.

[3] R.S. Beniwal and R. Singh, Calculation of thermal efficiency of salt-gradient solar ponds, Heat Recov. Syst. CHP, 7 (6), 497-516, 1987.

[4] T.L. Bergman, F.P. Incropera and R. Viskanta. A multi-layer model for mixing layer development in a double diffusive thermohaline system heated from below, Int. J. Heat Mass Transf. 25, 1411-1418, 1982.

[5] F. Bernad, S. Casas, O. Gibert, A. Akbarzadeh, J.L. Cortina and C. Valderrama, Salinity gradient solar pond: Validation and simulation model, Sol. Energy 98, 366374, 2013.

[6] R. Boudhiaf, A.B. Moussa and M. Baccar, A two-dimensional numerical study of hydrodynamic, heat and mass transfer and stability in a salt gradient solar pond, Energies 5 (12), 3986-4007, 2012.

[7] G. Boyle, Renewable Energy: Power for a Sustainable Future, 2nd ed., Oxford, UK: Oxford University Press, 2004.

[8] M.M. Dah, Etude numerique et experimentale de la stabilité des etangs solaires a gradient de sel, PhD. Thesis, University of Tunisia El Manar, 2010.

[9] A. Defant, Physical Oceanography, Pergamon Press, Oxford, UK, 1961.

[10] D. Gonzalez, J. Amigo, S. Lorente, A. Bejan and F.Suarez, Constructal design of salt gradient solar pond fields, Int. J. Energy Res. 10, 1428-1446, 2016.

[11] A. Guesmia and N. Daili, Finite volume approximation of stationary Burgers equation, J. Anal. Appl. 6 (3), 179-193, 2008.

[12] A. Guesmia and N. Daili, Approche numérique de la solution entropique de l'équation d'évolution de Bürgers par la méthode des lignes, Gen. Math. Sci. 17 (2), 99-111, 2009.

[13] A. Guesmia and N. Daili, Numerical approximation of fractional Burgers equation, Commun. Math. Appl. 1 (3), 1-16, 2010.

[14] J.R. Hull, C.E. Nielsen and P. Golding, Salinity-gradient solar ponds, CRC Press, Boca Raton, FL, 1989.

[15] P.D. Lax and R.D. Richtmyer, Survey of the stability of linear finite difference equations, Comm. Pure Appl. Math. 9, 267-293, 1956.

[16] M.M. Ould Dah, M.Ouni, A. Guizani and A. Belghith, The influence of the heat extraction mode on the performance and stability of a mini solar pond, Appl. Energy 87, 3005-3010, 2010.

[17] A. Rabl and C.E. Nielson, Solar ponds for space heating, Sol. Energy 17, 1-12, 1975.

[18] K.R. Sreenivas, J.H. Arakeri and J. Srinivasan, Modeling of the dynamics of the mixed layer in solar ponds, Sol. Energy 54 (3), 193-202, 1995.

[19] M. Turkyilmazoglu, An effective approach for approximate analytical solutions of the damped Duffing equation, Phys. Scr. 86, 015301, 2012.

[20] M. Turkyilmazoglu, Is homotopy perturbation method the traditional Taylor series expansion, Hacet. J. Math. Stat. 44 (3), 651-657, 2015.

[21] M. Turkyilmazoglu, Convergence accelerating in the homotopy analysis method: a new approach, Adv. Appl. Math. Mech. 10 (4), 925-947, 2018.

[22] M. Turkyilmazoglu, A simple algorithm for high order Newton iteration formulae and some new variants, Hacet. J. Math. Stat. 49 (1), 425-438, 2020.

[23] Y.F. Wang and A.A. Akbarzadeh, A parametric study on solar ponds, Sol. Energy 30, 555-562, 1983. 
[24] H. Wang, M. Xie and W. Sun, Nonlinear dynamic behavior of non-convective zone in salt gradient solar pond, Sol. Energy 85, 1745-1757, 2011.

[25] H. Xu, Laboratory studies on dynamical process in salinity gradient solar pond, Ph.D. Thesis, Ohio State University, 1990.

[26] F. Zangrando and H.J.S. Femando, A predictive model for migration of doublediffusive interfaces, Sol. Energy 113, 59-65, 1991. 